\title{
A One-Sided Competition Mathematical Model for the Sterile Insect Technique
}

\author{
Anis Ben Dhahbi, ${ }^{1,2}$ Yassine Chargui, ${ }^{1,2}$ Salah Mahmoud Boulaaras $\mathbb{D}^{3,4}$ \\ and Sana Ben Khalifa $\mathbb{D}^{1,5}$ \\ ${ }^{1}$ Department of Physics, College of Science and Arts at Ar Rass, Qassim University, P.O. Box 53, Ar Rass 51921, Saudi Arabia \\ ${ }^{2}$ Tunis El Manar University, Faculty of Sciences of Tunis, 2092 Tunis, Tunisia \\ ${ }^{3}$ Department of Mathematics, College of Science and Arts at Ar Rass, Qassim University, P.O. Box 53, \\ Ar Rass 51921, Saudi Arabia \\ ${ }^{4}$ Laboratory of Fundamental and Applied Mathematics of Oran (LMFAO) University of Oran Ahmed Benbella, Oran, Algeria \\ ${ }^{5}$ Laboratory of Energy and Materials (LabEM), ESSTHS, University of Sousse, $4011 \mathrm{H}$. Sousse, Tunisia
}

Correspondence should be addressed to Sana Ben Khalifa; sana.benkhalifa19@gmail.com

Received 6 June 2020; Accepted 8 July 2020; Published 30 July 2020

Guest Editor: Karthikeyan Rajagopal

\begin{abstract}
Copyright (c) 2020 Anis Ben Dhahbi et al. This is an open access article distributed under the Creative Commons Attribution License, which permits unrestricted use, distribution, and reproduction in any medium, provided the original work is properly cited.

We study a simple mathematical model describing the dynamics of a wild-type pest insects population experiencing competition from sterile insects (one-sided competition). This model can be used for conceiving control strategies based on the Sterile Insect Technique (SIT) or the Incompatible Insect Technique (IIT), aiming to reduce or eradicate Red Palm Weevil (RPW) populations in some target regions. We show that suppression may occur for continuous and periodic release strategies for various intraspecific and interspecific submodels except in the case of a single release strategy where a strong Allee effect is required.
\end{abstract}

\section{Introduction}

Over the past few decades, there has been an increasing interest in the effectiveness of the biological methods for the control of insect pests. The aim is to replace the existing insecticide-based control strategies. The SIT is a tool that has proven to be effective in the field for the areawide control of some insects. This method, suggested initially by Knipling $[1,2]$ and collaborators, relies on the release of a large number of sterilized males, without affecting their ability to mate. These released insects compete for mates with the wild-type males, and any wild-type female inseminated by a sterile male has no progeny. Then, if sufficient sterile males are released for a long enough period, most of the wild-type females in the field mate with the released sterile males and thus produce no viable offspring. The average fertility of the target population could be reduced leading to the control or even in certain situations to the eradication of the pest insects from large areas. The first successful areawide SIT programs have been operated against the screwworm population in Florida in the early 1950s. Since then, the technique has been applied to combat various agricultural pests and disease vectors, such as the Mediterranean fruit fly (medfly), the RPW in coconut and date palm gardens, and the tsetse fly in Africa (see Ref. [3] for a general review of the SIT and its applications). Given the experienced efficiency of the SIT, it is now believed by population ecologists that this tool should be included as a component of large programs of pest insects management.

Furthermore, other biocontrol methods, with similar acting principles as the classical SIT, are being developed. Particularly, we mention the IIT and Population replacement (PR). These two techniques utilize the Cytoplasmic Incompatibility (CI) property of the Wolbachia bacterium. This bacteria is known to infect various Arthropods, among them the RPW. Then, since Wolbachia-infected males (Wmales) are not able to fertilize uninfected eggs, due to CI, the massive releases of $\mathrm{W}$-males act like the classical SIT. On the 
other hand, the CI-property increases the progeny of $\mathrm{W}$-females considerably. Thus, as Wolbachia is maternally inherited, releasing a large number of $\mathrm{W}$-females into a target population may lead to a high abundance of Wolbachia-infected insects (population replacement), as was observed, for instance, with the Californian Culex pipiens [4]. This will eventually result in the elimination or the reduction of the pest population [5].

In view of the above considerations, many works have been oriented particularly to theoretical studies, especially of the SIT and the IIT, using mathematical modelling (see, for example, [5-12] and references therein). The main objectives are the understanding and the analysis of suppression mechanisms as well as the knowledge of explicit mathematical conditions for the total extinction of the target pests population. This may be very crucial to a SIT/IIT programme, since it permits to estimate its cost as it highlights the features to be considered when taking decision about strategies to adopt. Thus, various classes of models have been applied to these techniques, including deterministic, stochastic, continuous-time, discrete-time, and hybrid approaches (see Ref. [13] for a discussion of the relative merits of different models). The virtues of analytic models are their generality and the precision with which the actions of different parameters of the strategy can be predicted. On the other hand, their major shortcoming is the simplification which must be allowed to make the models mathematically tractable.

In this paper, we consider a simplified, but fairly reasonable, mathematical model for the extermination process using the SIT/IIT. As will be argued later, we assume the sterile-fertile interaction to be a one-sided competition that solely affects the fertile population negatively. This scenario is mathematically implemented by modifying the interspecific competition term in a recent predator-predator model for SIT [14]. Then, using an approximate averaging procedure, we derive analytically the mathematical conditions necessary to achieve the eradication of the wild population in finite time. In particular, relations between the extinction time, the size of the released sterile males, and model's parameters are explicitly obtained. Next, we compare our analytical findings with exact numerical results in order to assess the accuracy of the used approximations.

This paper is organized as follows. In Section 2, we present and argue our general mathematical model for the interactive dynamics between wild and sterile insects. In Sections 3 and 4, this model is used to approximate the conditions analytically to achieve the total extinction of the target population for different release strategies of sterile individuals. The obtained results are then discussed after being confronted with predictions of exact numerical calculations. In Section 5, we discuss the limitations and weaknesses of the considered model. In the last section, we give our conclusion.

\section{Model Formulation}

2.1. Assumptions and Equation Dynamics. The model considered in this work assumes continuous-time, deterministic and density-dependent growth of pest insects [15]. We also adopt most of the general assumptions, which apply to biological situations, originally discussed in [15]. Moreover, we assume that released sterile individuals are male-only and that all sorts of external factors, such as migration, environmental factors, mutation, and selection play no role. In addition, to keep the model simple, we do not include any stage distinction (one-stage model). Furthermore, it is agreed that, for many insects, most of the density dependence occurs in larval survivorship as a result of competition for natural resources [16]. Hence, we argue that sterile-fertile competition is a one-sided competition. Indeed it is widely admitted that SIT emphasis the competition for mates rather than for natural resources. Such competition obviously affects only the fertile population. Conversely, the sterile population is not affected by the presence of fertile individuals neither through the competition for mates (sterile individuals) nor through the competition for natural resources (no larval survivorship in the field). It is why we think that predator-predator and cannibalism models are not convenient for SIT modelling. Thus, we shall assume henceforth a one-sided competition affecting wild-type insects solely.

Mathematically, the model for these two species is described by a one-stage nonlinear dynamic system whose properties appear to depend essentially on the form of the growth rate of a native wild-type population $N(t)$, and their interaction with the sterile individuals of density $M(t)$. Consider the following density-dependent evolution equations subject to one-sided competition:

$$
\begin{aligned}
& \frac{\mathrm{d} M}{\mathrm{~d} t}=-\frac{1}{\tau_{s}} M+m_{R}, \quad t \geq 0, \\
& \frac{\mathrm{d} N}{\mathrm{~d} t}=r N F(N)-\delta M H(N), \quad t \geq 0,
\end{aligned}
$$

where $m_{R}$ is a function of time characterizing the release strategy of sterile males whose average life-time is $\tau_{s}$. The term $r F(N)$ is the per-capita growth rate of wild-type population. The parameter $r$ allows us to scale, if needed, the continuous function $F(N)$ so that $\max _{N} F(N)=1$. In that case, $r$ is the maximum per-capita growth rate without any interference from other individuals in the population. The density dependence of adult pest insects growth rate can be seen as a remanent feature of natural resource limitation at the larval stage. The interaction term $\delta M H(N)$ describes the sterile-fertile one-sided competition where $H(N)$ is the functional response and the parameter $\delta$ is the interaction rate between the two species. Note that the death rate of the wild-type population has been ignored in equation (2).

2.2. Intraspecific and Interspecific Competitions. The choice of the intraspecific competition model appears to condition the population dynamics. While early studies tended to assume the population growth rate to be logistic, more recently, much attention has been paid to the impact of the Allee effect, as the latter was shown to affect virtually all aspects of species interactions in space and time $[17,18]$. 
This negative competition effect refers to a decrease in the per-capita growth rate at low population densities, which may increase the extinction risk of low-density populations $[19,20]$. This effect arises from a number of sources such as difficulties in finding mates, social dysfunction, and inbreeding depression [21] and may have potential importance in the dynamics of small populations $[22,23]$, as well as in population diffusion processes [24-27].

Let us assume that the wild-type population has a stable steady state $F\left(N_{s}^{h}\right)=0$ : that is, the carrying capacity of the niche in the absence of sterile males. When the Allee effect is sufficiently strong, there is also an unstable steady density $N_{u}^{l}$ of wild-type insects, such that $0<N_{u}^{l}<N_{s}^{h}$, and for which $F\left(N_{u}^{l}\right)=0$. These states are given in Table 1 , for the two types of function $F(N)$ that we are going to consider in this work.

For $F_{2}$ the submodel, the condition $0<\alpha<\beta$ ensures a strong Allee effect [24]. Thus, the parameter $\alpha$ can be defined as "Allee effect constant"; the bigger $\alpha$ is, the stronger the Allee effect will be.

On the other hand, for interspecific submodels, describing the one-sided competition between sterile and fertile populations, we shall consider functional responses of Holling type in addition to a flat mating rate response. The latter are shown in Table 2. For Holling type-II and type-III responses, the parameter $s$ measures the "satiation" effect: the mating with fertile wild-type females by a unit number of a sterile male cannot continue to grow linearly with the number of wild-type females available but must "saturate" at the value $1 / s$ (see for instance $[28,29]$ ). If the number of available male matings equals or exceeds the number of available female matings, then all available matings with fertile females occur. Besides, as long as mating is random, the proportion of fertile female matings to fertile and sterile males will not be altered by the presence of females that were fecundated. It is obvious that this submodels are not equivalent and some criteria based on field experiments and state-of-the-art for SIT should be considered when drawing conclusions regarding their applicability.

\section{Extinction Condition}

Regarding the cost and the duration of SIT, it is crucial to have information on the underlying factors of the Threshold Amount of Sterile Males (TASM) to be released in order to achieve total extinction. Our procedure will provide a good estimation of TASM for various competition submodels and release strategies, by relating the condition for total extinction to the initial number, $M_{\text {init }}$, of sterile individuals released in the niche.

Firstly, it is assumed that insect population density is initially in a stable steady state. Then, to take into account the eventual Allee effect, we merely have to reduce the pest population density from $\beta$ to $\alpha_{\text {sup }}$ in order to achieve extinction. In practical terms, this requires that interspecific competition should be stronger than the intraspecific competition as $N$ goes from $\beta$ to $\alpha_{\text {sup }}$ in the typical time scale $\tau_{e}$ of a given release strategy. Furthermore, by integrating equation (2) between 0 and $\tau_{e}$ we get the following:
TABLE 1: Intraspecific growth submodels of wild-type insect growth rate.

\begin{tabular}{lcc}
\hline$F(N)$ & $N_{s}^{h}$ & $N_{u}^{l}$ \\
\hline$F_{1}(N)=1-(N / \beta)$ & $\beta$ & 0 \\
$F_{2}(N)=k_{2}(1-(N / \beta))(N /(\gamma+N)), \gamma>0$ & $\beta$ & 0 \\
$F_{3}(N)=k_{3}(\alpha-N)(N-\beta), 0<\alpha<\beta$ & $\beta$ & $\alpha$ \\
\hline
\end{tabular}

$N_{s}^{h}$ and $N_{u}^{l}$ stand, respectively, for the highest stable steady state and the lowest unstable one. To meet $\max _{N} F=1$ condition, the normalization constants should be set at $k_{2}=\beta /(2 \gamma+\beta-2 \sqrt{\gamma(\gamma+\beta)})$ and $k_{3}=4 /(\alpha-\beta)^{2}$.

TABLE 2: Interspecific models of sterile-fertile competition.

\begin{tabular}{lc}
\hline$H(N)$ & Submodel \\
\hline$H_{I}(N)=N$ & Holling type-I \\
$H_{\text {II }}(N)=N /(1+s N)$ & Holling type-II \\
$H_{\text {III }}(N)=N^{2} /\left(1+s N^{2}\right)$ & Holling type-III \\
$H_{\text {IV }}(N)=1 / s$ & Flat mating rate \\
\hline
\end{tabular}

The parameter $s$ is a positive real number.

$$
N\left(\tau_{e}\right)=\beta+\int_{0}^{\tau_{e}}[r N F(N)-\delta M H(N)] \mathrm{d} t .
$$

The extinction condition, $N\left(\tau_{e}\right) \leq \alpha_{\text {sup }}$, leads then to the following equation:

$$
r\langle N F(N)\rangle-\delta\langle M H(N)\rangle \leq-\frac{\left(\beta-\alpha_{\text {sup }}\right)}{\tau_{e}},
$$

where the symbol $\langle\cdot\rangle$ denotes the time average over the interval $\left(0, \tau_{e}\right)$. Then, equation (4) can be rewritten as follows:

$$
\langle M H\rangle \geq \frac{1}{\delta}\left[r\langle N F\rangle+\frac{\left(\beta-\alpha_{\text {sup }}\right)}{\tau_{e}}\right] .
$$

Since $H(N(t))$ and $M(t)$ are measurable functions over $\left(0, \tau_{e}\right)$, then it is possible to use Hölder's inequality, according to which we have the following equation:

$$
\langle M H\rangle \leq\left\langle M^{p}\right\rangle^{1 / p}\left\langle H^{q}\right\rangle^{1 / q},
$$

where $p, q \in] 1,+\infty[$ and $1 / p+1 / q=1$. This yields a more stringent extinction condition, given by the following equation:

$$
\left\langle M^{p}\right\rangle^{1 / p} \geq \frac{1}{\delta\left\langle H^{q}\right\rangle^{1 / q}}\left[r\langle N F\rangle+\frac{\left(\beta-\alpha_{\text {sup }}\right)}{\tau_{e}}\right] .
$$

The last formula summarizes the effects of each term in equation (2) on the minimum size of the sterile males to be released. Moreover, a key component of our approach is to replace the time-averaged functionals of $N(t)$ in equation (7), $\left\langle H^{q}\right\rangle$ and $\langle N F\rangle$, by their values on $\langle N\rangle$, and to adopt a linear approximation for $\langle N\rangle$, that is,

$$
\langle N\rangle \approx \frac{\alpha_{\text {sup }}+\beta}{2} \text {. }
$$

These approximations should naturally result in some errors, and we shall rely on numerical simulations to check 
their validity. On the other hand, the adopted approximation of the averaged functionals of $N(t)$ removes the dependence of equation (7) on the parameter $q$, and leaves us a free choice of parameter $p$. Later, the value of $p$ will be chosen so as to get the best fit for numerical results. Tables 3 and 4 give $\langle N F(N)\rangle$ and $\langle H(N)\rangle$ for various models of growth rate and sterile-fertile interaction.

\section{Mass Release Strategies}

A given control strategy may succeed or fail depending largely not only on various ecological characteristics of the target species but also on the choice of the mass release strategy of sterile males. In order to investigate the parameters that govern the effectiveness of SIT control strategies, in what follows, we apply the above results for three different strategies for the release of sterile males, which are the single release, the constant release, and the periodic release.

4.1. Single Mass Release. Although a single release strategy may be seen as an unual practice, its investigation could highlight relevant parameters to be considered for other release strategies. For single mass release, the sterile population density follows the law:

$$
\frac{\mathrm{d} M}{\mathrm{~d} t}=-\frac{1}{\tau_{s}} M
$$

so that $M(t)=M_{\text {init }} e^{-t / \tau_{s}}$ where $\tau_{s}$ is the sterile insects average life-time and $M_{\text {init }}$ is the initially released number. For this strategy, the sterile males are gone after the release generation and have no more effect. Therefore, the wild-type population is expected to decrease under the SIT effect within a time scale $\tau_{e}$ of the order of $\tau_{s}$. An interesting result is that for a single release strategy, extinction does not occur for $F_{1}$ and $F_{2}$, for all Holling type submodels considered in this work. Indeed, for a weak Allee effect, the condition (4) requiring an effective negative growth rate that could not be satisfied as $\Delta t \longrightarrow+\infty$. Because of the exponentially decreasing effect of sterile insects, there exits a time $t_{0}$ after which the wild population recovers persistent positive growth rate until it reaches its highest stable steady state again. This result is well proved by numerical calculations as illustrated in Figure 1, which displays the behavior of the wild-type population $N(t)$ corresponding to $F_{1}$ and $F_{2}$ for interspecific submodels $H_{\mathrm{I}}, H_{\mathrm{II}}$, and $H_{\mathrm{III}}$.

Then, only a strong Allee effect allows for effective extinction when the single release strategy is chosen. In this case, we have the following:

$$
\left\langle M^{p}(t)\right\rangle^{1 / p}=\left[\frac{\tau_{s}}{p \tau_{e}}\left(1-e^{-p \tau_{e} / \tau_{s}}\right)\right]^{1 / p} .
$$

TABLE 3: Average factors for various intraspecific models of wildtype insect growth rate.

\begin{tabular}{lc}
\hline$F(N)$ & $\langle N F\rangle$ \\
\hline$F_{1}(N)$ & $\beta / 4$ \\
$F_{2}(N)$ & $\beta^{3} / 4(2 \gamma+\beta)(2 \gamma+\beta-2 \sqrt{\gamma(\gamma+\beta)})$ \\
$F_{3}(N)$ & $(\beta+\alpha) / 2$ \\
\hline
\end{tabular}

TABLE 4: Average factors for various interspecific models of sterilefertile competition.

\begin{tabular}{lc}
\hline$H(N)$ & $\langle H\rangle$ \\
\hline$H_{\mathrm{I}}(N)$ & $\left(\beta+\alpha_{\text {sup }}\right) / 2$ \\
$H_{\text {II }}(N)$ & $\left(\beta+\alpha_{\text {sup }}\right) / 2+s\left(\beta+\alpha_{\text {sup }}\right)$ \\
$H_{\text {III }}(N)$ & $\left(\beta+\alpha_{\text {sup }}\right)^{2} / 4+s\left(\beta+\alpha_{\text {sup }}\right)^{2}$ \\
$H_{\text {IV }}(N)$ & $1 / s$ \\
\hline
\end{tabular}

Hence, according to equation (7), for the $F_{3}$ submodel, the TASM to achieve effective extinction in population size with a single release, within a period of time $\tau_{e}$, is given by the following equation:

$$
M_{\mathrm{TASM}}=\left(\frac{p \tau_{e} e^{p \tau_{e} / \tau_{s}}}{\tau_{s}\left(e^{p \tau_{e} / \tau_{s}}-1\right)}\right)^{1 / p} \frac{1}{\delta\langle H\rangle}\left[\frac{r}{2}(\beta+\alpha)+\frac{\beta-\alpha}{\tau_{e}}\right] .
$$

Before proceeding further, we would like to make some comments on Flores's work [14]. Flores used a model with $H_{\mathrm{I}}$ and a nonnormalized $F_{3},\left(k_{3}=1\right)$, with $r=1$ and assuming $\tau_{e}=\tau_{s}$ which should yield the following equation:

$$
M_{\mathrm{TASM}}=\left(\frac{p e^{p}}{e^{p}-1}\right)^{1 / p} \frac{(\beta-\alpha)}{\delta(\beta+\alpha)}\left[\frac{2}{\tau_{s}}+\frac{\left(\beta^{2}-\alpha^{2}\right)}{4}\right] .
$$

It is worth emphasizing here that Flores's result, quoted in [14], does not show any dependence on $\tau_{s}$. Numerical simulations agree with the above result for a large range of parameters as shown in Figure 2. In particular, we find that numerical $M_{\text {TASM }}$ exhibit an explicit dependence on the lifetime of sterile insects corroborating our claim that results in [14] were incomplete.

For an empirical investigation of this result, let us consider the case where, $\beta \gg \alpha$, we get for the same assumptions:

$$
M_{\mathrm{TASM}}=\frac{1}{\delta}\left(\frac{p e^{p}}{e^{p}-1}\right)^{1 / p}\left[\frac{1}{\tau_{s}}+\frac{1}{8} \beta^{2}\right] .
$$

It is interesting to notice that for this choice, the minimum sterile insect amount to release goes like $\beta^{2}$. This is counter-intuitive if we learn that for entomologists its is widely assumed that $M_{\mathrm{TASM}}$ should go like $\beta$ : constant ratio behavior. This shortcoming is due in part to the fact that $F_{3}$ is 


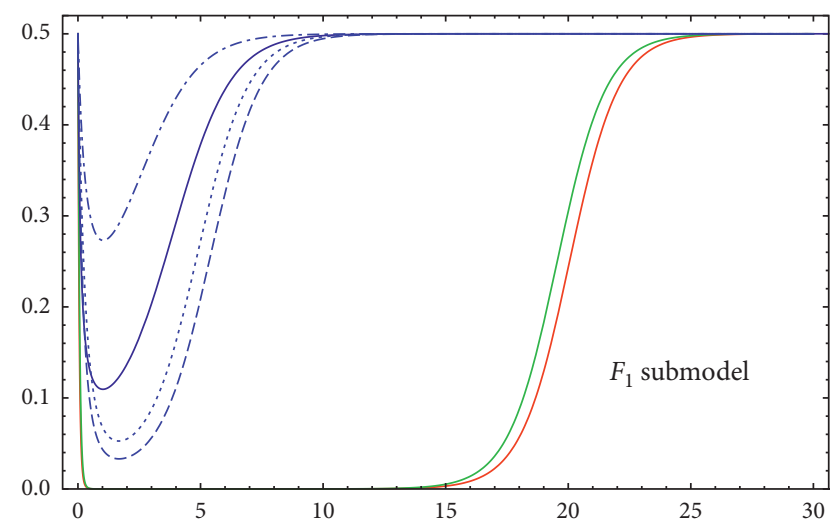

(a)

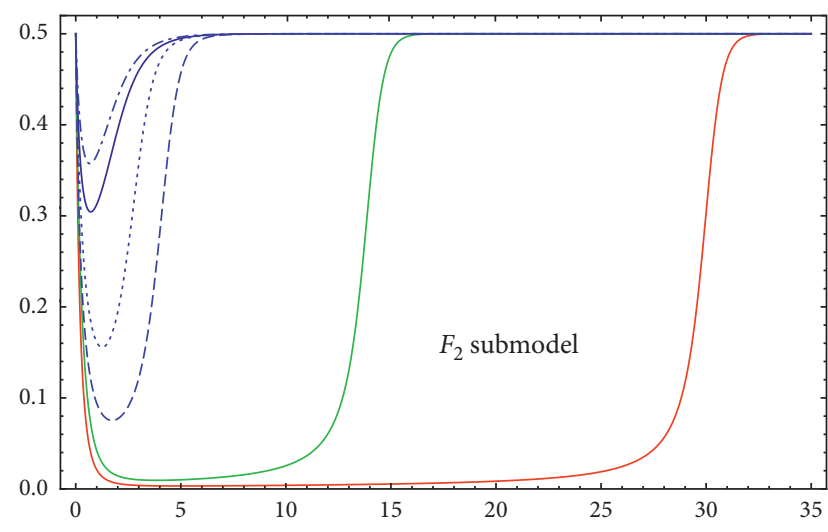

(b)

Figure 1: Plots of $N(t)$ for single release strategy in cases of $F_{1}$ and $F_{2}$ for interspecific models $H_{\mathrm{I}}, H_{\mathrm{II}}$, and $H_{\mathrm{III}}$. Solid (broken) lines correspond to $M_{\text {init }}=20\left(M_{\text {init }}=5\right)$ in the case of $F_{1}$ and to $M_{\text {init }}=6\left(M_{\text {init }}=4\right)$ in the case of $F_{2}$.

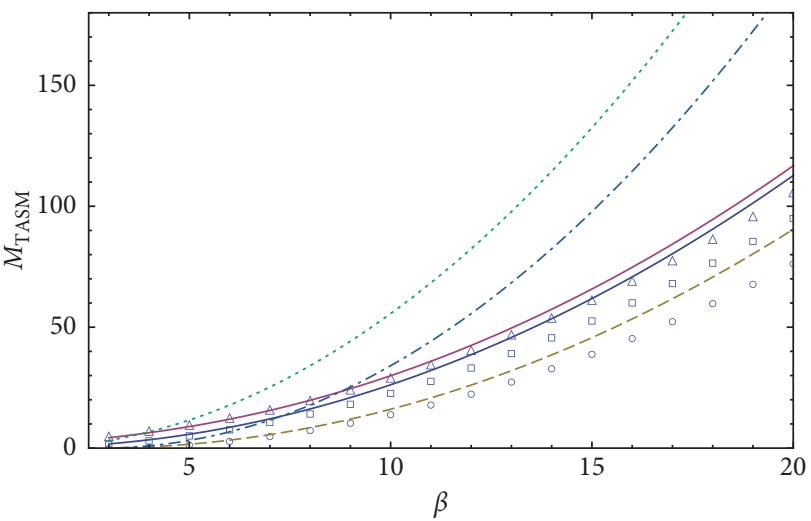

FIGURE 2: Theoretical and simulated $M_{\text {TASM }}$ as a function of $\beta$. Our (Flores) theoretical result is represented by thick (dotted) line (for $\tau_{s}=5$ and $\alpha=0.8$ ), thin (dotted) line (for $\tau_{s}=0.5$ and $\alpha=0.8$ ), and dashed (dot-dashed) line (for $\tau_{s}=5$ and $\alpha=2.8$ ). Rectangles, triangles, and circles correspond to simulated results for the same choices of parameters $\tau_{s}$ and $\alpha$, respectively. The remaining parameters values are $\delta=1$ and $p=13$.

not normalized. On the other hand, it is quite interesting to see that for a normalized growth rate, the constant ratio behavior of the TASM can only be obtained through the submodel of interspecific competition. This requires $H(N)$ to be a slowly varying function which is achieved for $H_{\mathrm{II}}$, $H_{\mathrm{III}}$, and $H_{\mathrm{IV}}$. The work on our assumptions leads here to the following:

$$
M_{\mathrm{TASM}}=\frac{1}{\delta\langle\widehat{H}\rangle}\left(\frac{p e^{p}}{e^{p}-1}\right)^{1 / p}\left[r+\frac{2}{\tau_{s}} \frac{(\beta-\alpha)}{(\beta+\alpha)}\right] .
$$

On the other hand, for $\beta \gg \alpha$, we have $\left\langle\widehat{H}_{\text {II-III-IV }}\right\rangle \simeq \beta / 2$. Therefore we get the following equation:

$$
M_{\mathrm{TASM}}=\frac{r \beta}{\delta}\left(\frac{p e^{p}}{e^{p}-1}\right)^{1 / p}\left[\frac{1}{r \tau_{s}}+\frac{1}{2}\right],
$$

proving that our assumptions allow us to obtain the empirically justified behavior. In the Flores model, symmetric competition between fertile and sterile insects seems to be related to the availability of resources. Although in finite niche, natural resources are limited, for adult insects, it is not a limiting factor. Limited resources are much more critical at juvenile stages (larval) when there is no fertile-sterile competition [30]. Therefore, fertile-sterile competition on resource need not affect a population's growth rate. Competition is strictly related to mating.

Note that more realistic models would be compliant with the empirical "sterile-to-fertile ratio" consideration that is the sterile-to-fertile males ratio is one of the most relevant parameters in SIT programmes [31].

To test the theoretical predictions presented above, evolution equation (2) was solved numerically using Mathematica 9. The initial wild-type population was supposed to be at its highest stable steady state. The numerical computations for the three submodels closely agree with the analytical result, as shown in Figure 3.

4.2. Continuous Mass Release. Multiple releases are usually necessary to eradicate target populations. A continuous release strategy is an idealization of what is done in practice. Here, we assume that the release of sterile males is performed uniformly in time with a rate $m$. Under this assumption, the population dynamics is governed by the following system:

$$
\frac{\mathrm{d} M}{\mathrm{~d} t}=-\frac{1}{\tau_{s}} M+m .
$$

Ignoring the transitory exponential decaying solution we have the following:

$$
M(t)=\tau_{s} m,
$$

yielding

$$
m_{\mathrm{TASM}}=\frac{1}{\tau_{s} \delta\langle\hat{H}\rangle}\left[r\langle F\rangle+\frac{2}{\tau_{e}} \frac{\left(\beta-\alpha_{\text {sup }}\right)}{\left(\beta+\alpha_{\text {sup }}\right)}\right] .
$$

In addition, as the continuous release strategy is based on a long-time scale: $\tau_{e} \gg 1$, the extinction condition becomes as follows: 


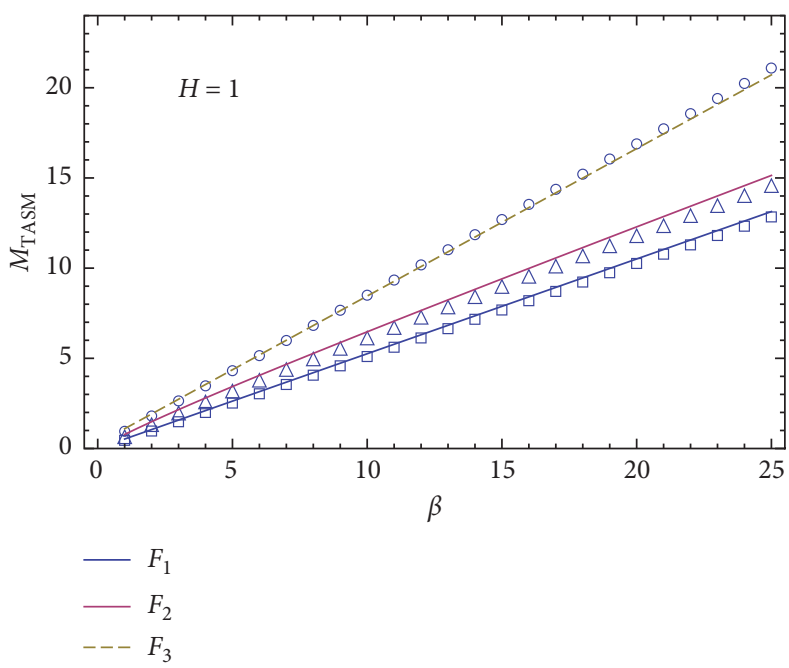

(a)

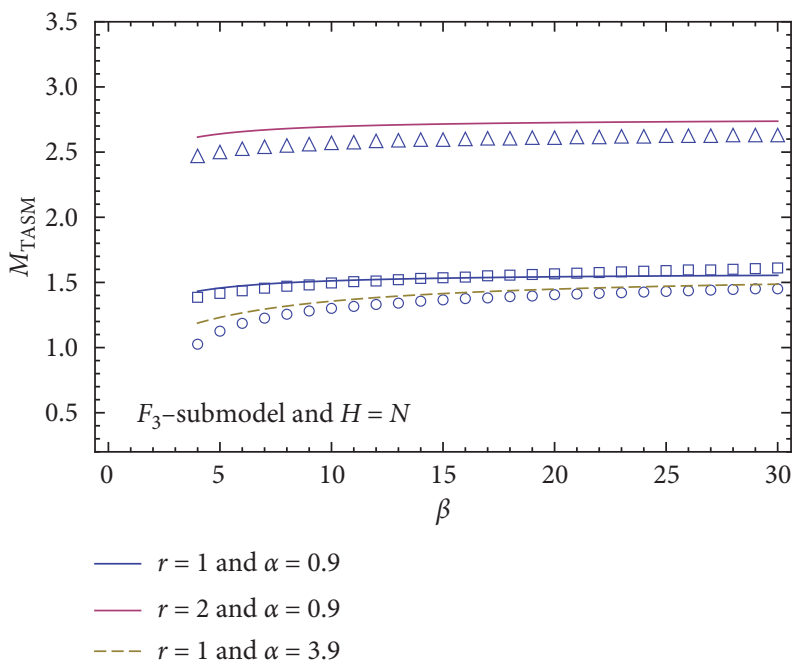

(c)

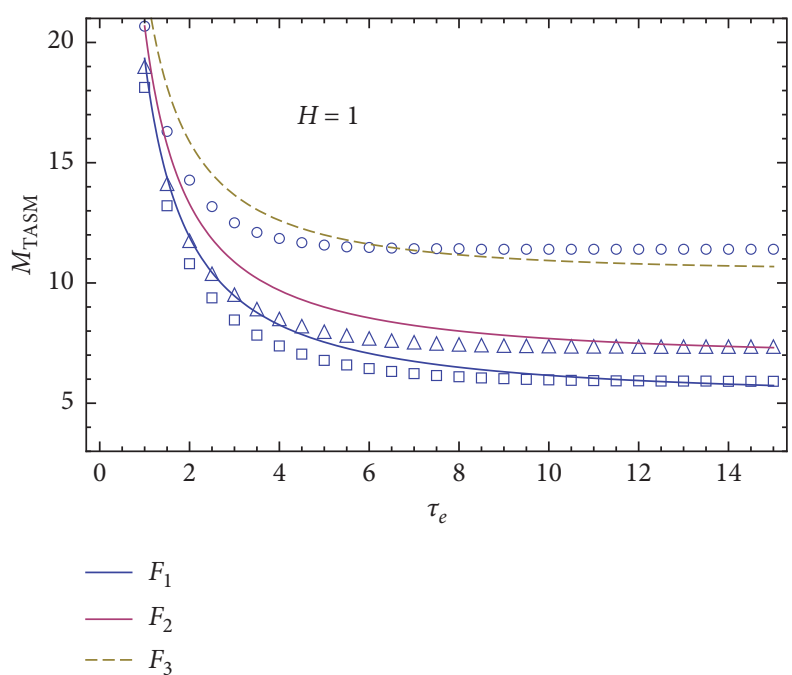

(e)

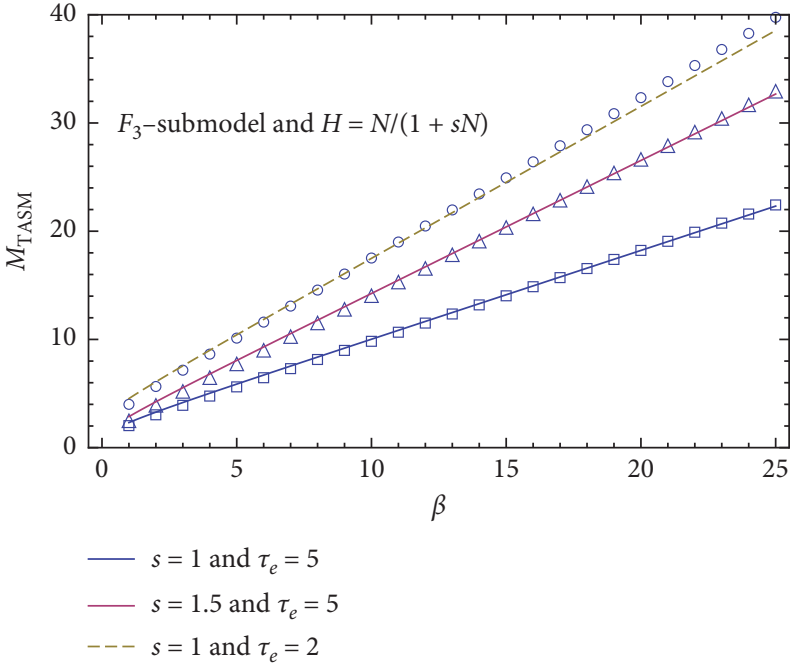

(b)

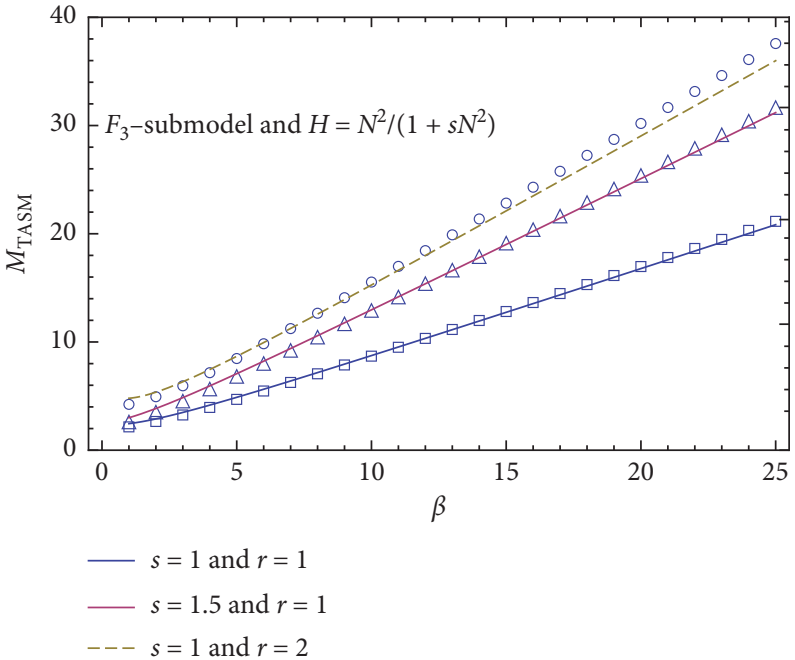

(d)

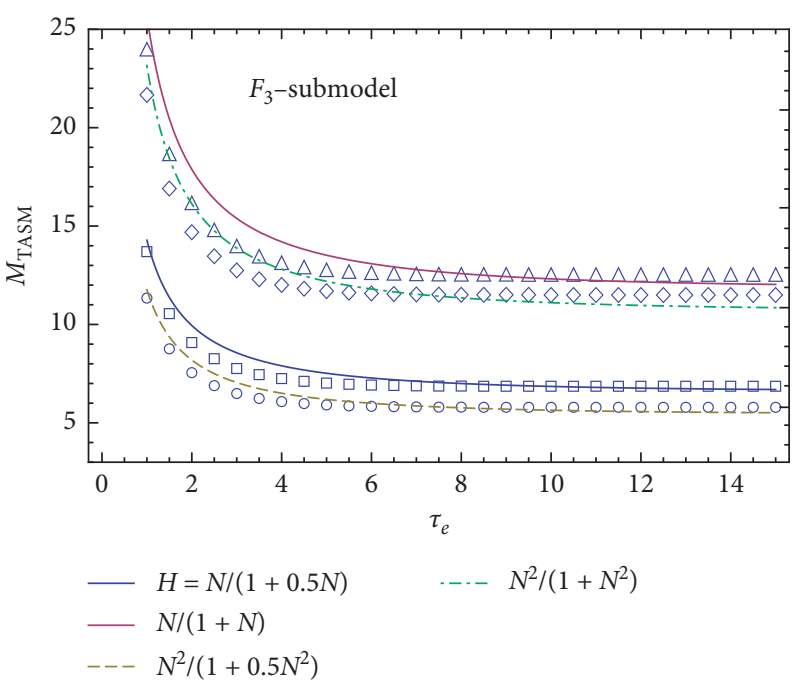

(f)

Figure 3: Theoretical and simulated $M_{\text {TASM }}$, in the case of single mass release strategy, as a function of $\beta$ and as a function of $\tau_{e}$. The remaining parameters values are $\delta=1, \gamma=0.5$, and $p=13$. (a) Parameters values are as follows: $r=1, \tau_{s}=8, \tau_{e}=5$, and $\alpha=0.8$, (b) parameters values are as follows: $r=1, \tau_{s}=8$, and $\alpha=0.8$, (c) parameters values are as follows: $\tau_{s}=8$ and $\tau_{e}=6$, (d) parameters values are: $\tau_{e}=5, \tau_{s}=8$, and $\alpha=0.8$, (e) parameters values are as follows: $r=1, \tau_{s}=15, \beta=15$, and $\alpha=0.8$, (f) parameters values are as follows: $r=1$, $\tau_{s}=15, \beta=15$, and $\alpha=0.8$. 


$$
m_{\mathrm{TASM}}=\frac{r}{\tau_{s} \delta} \frac{\langle F\rangle}{\langle\widehat{H}\rangle} .
$$

In particular, for all intraspecific and interspecific submodels, and for $\beta \gg \alpha$ we expect the following:

$$
m_{\mathrm{TASM}}=\frac{r \beta}{2 \tau_{s} \delta} .
$$

This result is of utmost interest as it clearly shows the relevant factors that should be considered for SIT. Numerical results summarized in Figure 4 show that all submodels closely agree with the theory.

4.3. Periodic Mass Release. A periodic release strategy is the most effective in what is done in practice. The sterile insect population dynamics goes as follows:

$$
\frac{\mathrm{d} M}{\mathrm{~d} t}=-\frac{1}{\tau_{s}} M+m(1+\varepsilon \cos (\omega t+\varphi)),
$$

where $\varepsilon$, $\omega$, and $\varphi$ are independent constants with $|\varepsilon| \leq 1$. Ignoring the exponentially decaying transitory solution we get the following:

$$
M(t)=\tau_{s} m\left(1+\frac{\varepsilon}{1+\tau_{s}^{2} \omega^{2}} \cos (\omega t+\varphi)+\frac{\varepsilon \tau_{s} \omega}{1+\tau_{s}^{2} \omega^{2}} \sin (\omega t+\varphi)\right) .
$$

The extinction dynamics depend on the relative time scales described by $\omega \tau_{s}$ and $\omega \tau_{e}$. Notice that in practice, the period of mass-releases is usually chosen to be close to the average life-time of sterile males, and the relative time scale $\omega \tau_{s}$ is so of the order of some units. Furthermore, the nature of this strategy implies that the extinction time $\tau_{e}$ is well above the average life-time $\tau_{s}$.

Next, for this strategy, we shall use the result (7) with $p=2$ (when Hölder's inequality reduces to Cauchy-Schwartz inequality). This yields the following extinction condition:

$$
m_{\mathrm{TASM}}=\left(1+\frac{\varepsilon^{2}}{2\left(1+\tau_{s}^{2} \omega^{2}\right)}\right)^{-1 / 2} \frac{1}{\tau_{s} \delta\langle\hat{H}\rangle}\left[r\langle F\rangle+\frac{2}{\tau_{e}} \frac{\left(\beta-\alpha_{\text {sup }}\right)}{\left(\beta+\alpha_{\text {sup }}\right)}\right],
$$

where we have neglected terms in $1 / \omega \tau_{e}$ in the calculation of $\left\langle M^{p}\right\rangle^{1 / p}$. We can see that for $\omega \tau_{s} \gg 1$ we recover the continuous release strategy result. Cases where $\omega \tau_{s} \ll 1$ yield the following:

$$
m_{\mathrm{TASM}}=\frac{1}{\tau_{s} \delta \sqrt{1+\left(\varepsilon^{2} / 2\right)}\langle\hat{H}\rangle}\left[r\langle F\rangle+\frac{2}{\tau_{e}} \frac{\left(\beta-\alpha_{\text {sup }}\right)}{\left(\beta+\alpha_{\text {sup }}\right)}\right],
$$

with respect to the first two cases, for this release strategy, several parameters should be taken into account, namely $\varepsilon$, $\omega$, and $\varphi$. This result is of utmost interest as it clearly shows the relevant factors that should be considered for SIT. Numerical results summarized in Figure 5 show that all submodels closely agree with the theory.

\section{Limitations of the Model}

In this section, we summarise the main weaknesses of this study. First, we are aware that the assumptions introduced in our model suffer from possible violations. Some of the more obvious are as follows:

(i) The hypothesis that the carrying capacity of a given environment is constant does not describe the real situation when the latter and thus the density-dependent death rate may vary between good and poor patches. Hence, the effect of time-varying carrying capacity should be investigated.

(ii) We have assumed a simple constant dispersal weevils rates, but unlikely, sterile males do not disperse uniformly, although releases in SIT programmes must be very heterogeneous over the treatment area. Weevils require food, mates, oviposition sites, and refugia as essential resources [32], foraging for these resources is a dynamic process. For instance, it has been observed that the RPW adjusts its activity in response to the change of weather factors and climatic conditions [33, 34]. Furthermore, human managements, like the application of pesticides or the presence of pheromone traps, also have an effect on the distribution of weevils.

(iii) Pests dispersal has been ignored; however, a more realistic model should take it into account. Host availability, susceptibility and its location in space are one of the major determinants of spatial distribution.

In addition, we have some objections to raise against the model, of which we will mention the two most important ones. First, the model does not account for time lags in the working of density on growth rate. In the RPW, for example, it may take several weeks or months before larvae develop into mature individuals $[35,36]$. The logistic model may be adequate in this respect for organisms with a simple life cycle but has to be modified for organisms with more complex life cycles. This has led to socalled time lag models (see, among others, $[37,38]$ ). These models show that such lags may lead to considerable fluctuations in the density of populations instead of stable equilibrium densities.

Second, remating in wild RPW females is common in nature; the probability that a wild female will remake after mating with a sterile male is highly relevant to the effectiveness of the SIT. The model considers the competition as related to mating but does not take into account the remating behavior. Sterile males are less able than wild males to suppress remating in wild females. This effect is increased in males that have been colonized for a longer time. Many factors are involved in the interaction between wild and sterile, sexual maturity synchronization, compatibility, etc., but further field data and investigations are needed to consider all these parameters and to incorporate them in the model. 

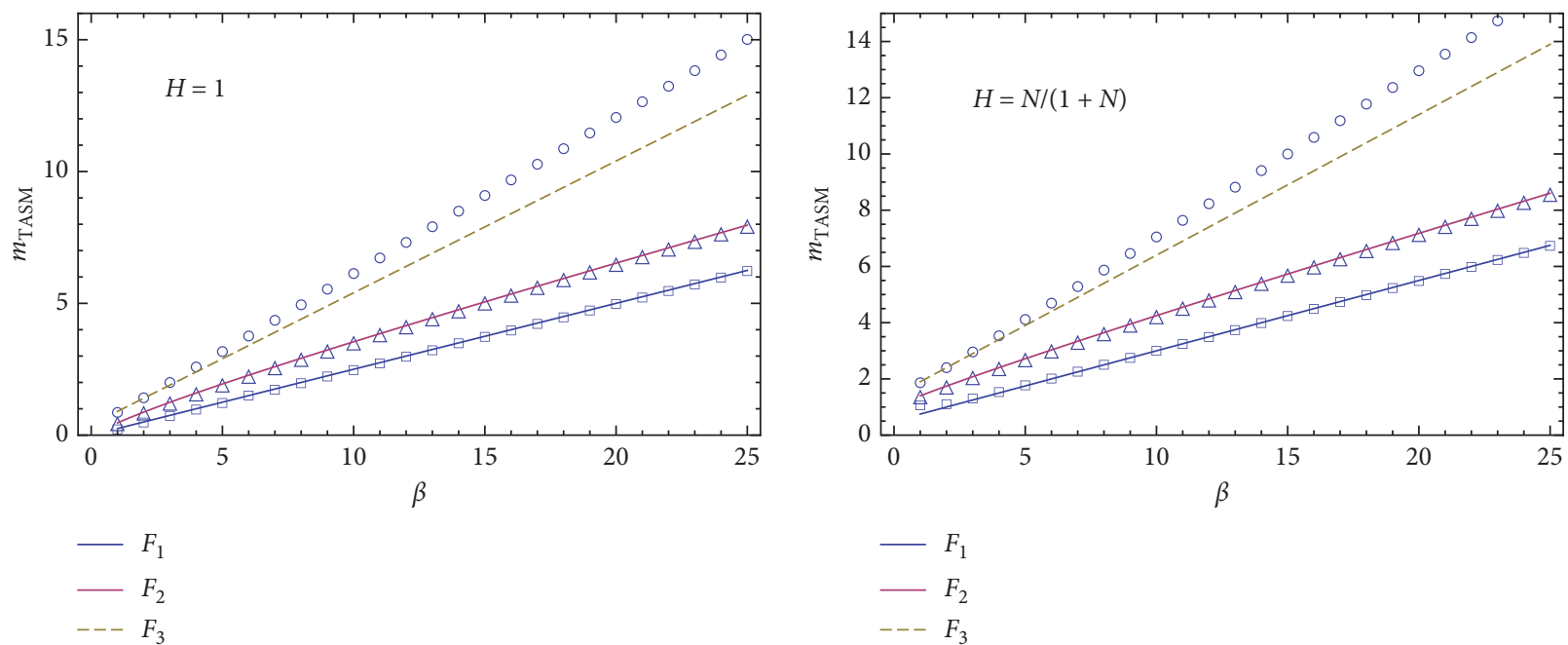

(a)

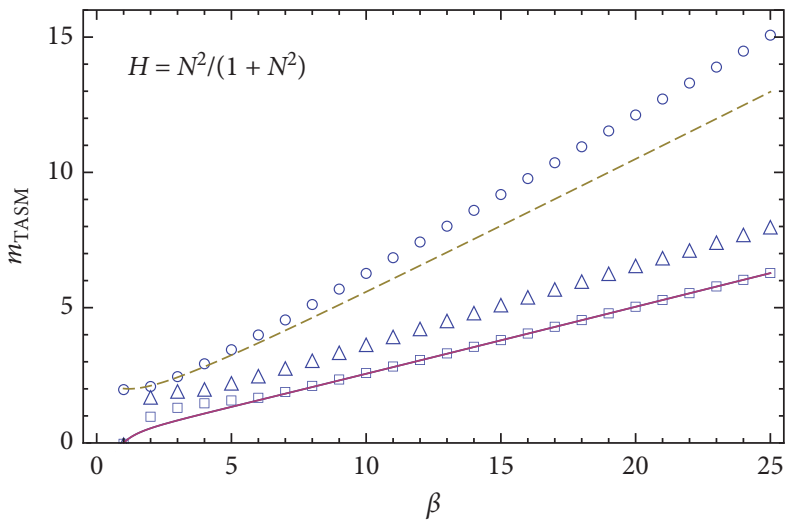

(b)

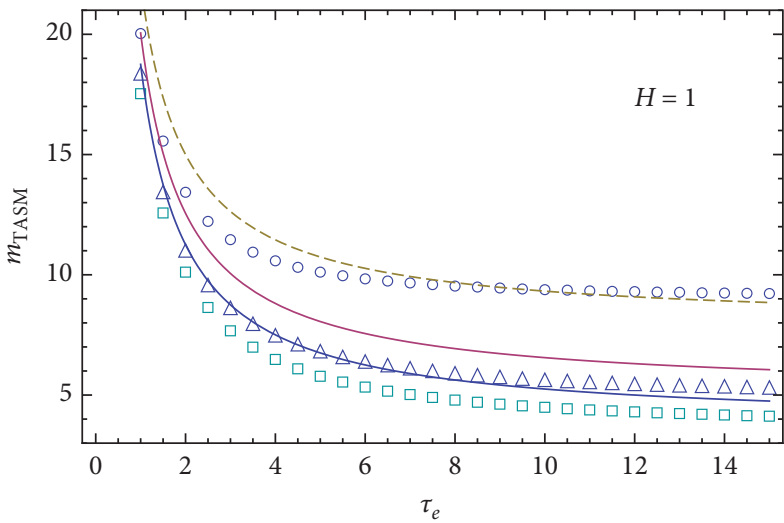

$\begin{array}{ll}- & F_{1} \\ - & F_{2} \\ --- & F_{3}\end{array}$

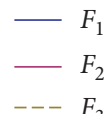

(c)

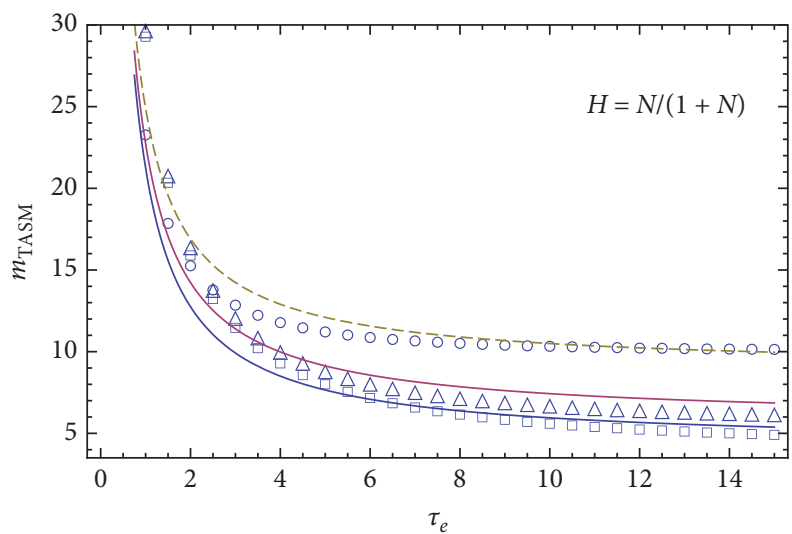

(d)

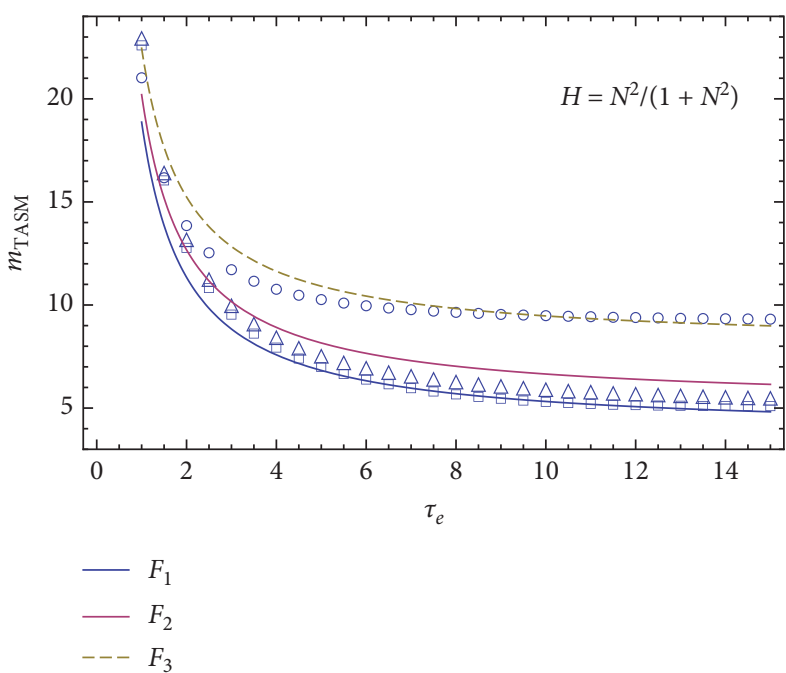

(e)

(f)

Figure 4: Theoretical and simulated $m_{\mathrm{TASM}}$, in the case of continuous mass release strategy, as a function of $\beta$ and as a function of $\tau_{e}$. Parameters values are $r=1, \delta=1, \tau_{s}=1, \alpha=0.8$, and $\gamma=0.5$. (a) Parameters values are $\tau_{e}=500$, (b) parameters values are $\tau_{e}=500$, (c) parameters values are $\tau_{e}=100$, (d) parameters values are $\beta=15$ (e) parameters values are $\beta=15$, and (f) parameters values are $\beta=15$. 


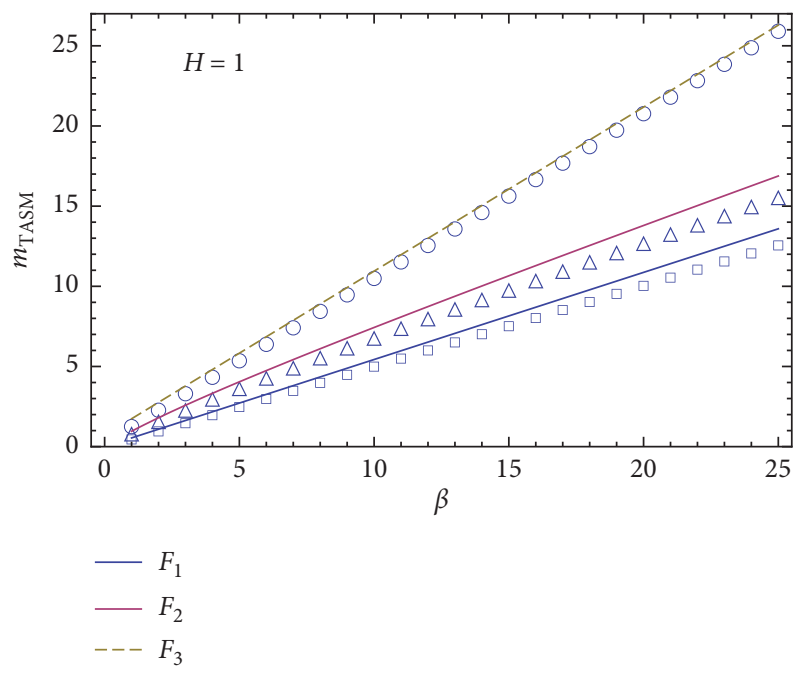

(a)

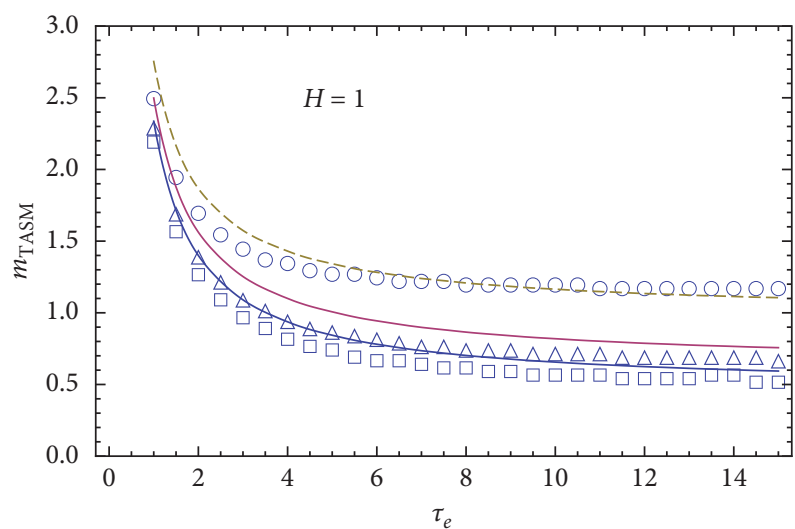

$$
\begin{aligned}
& -F_{1} \\
& -F_{2} \\
& ---F_{3}
\end{aligned}
$$

(c)

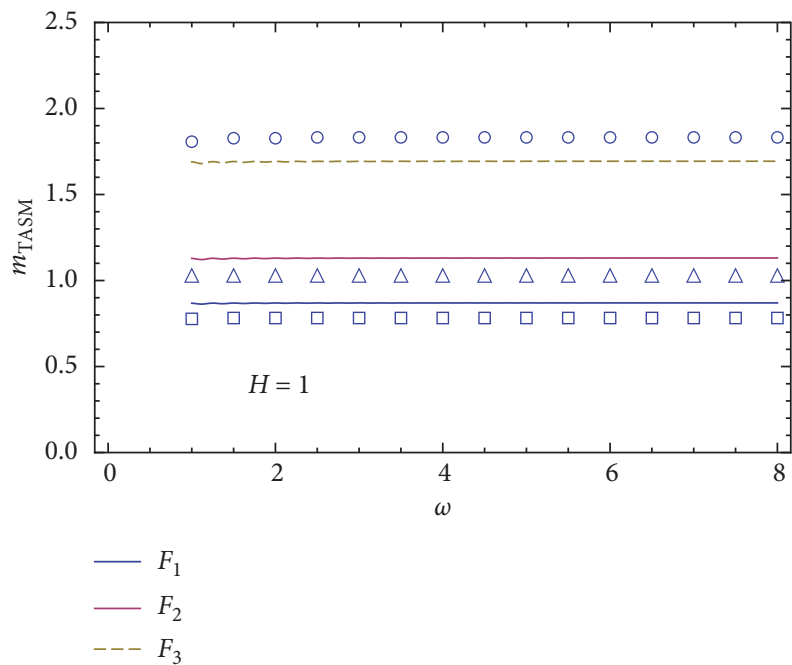

(e)

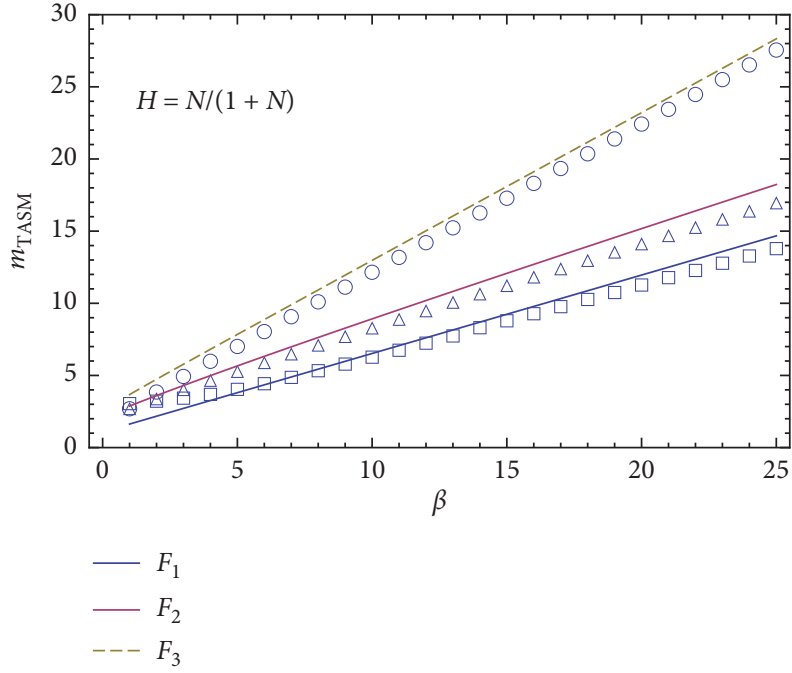

(b)

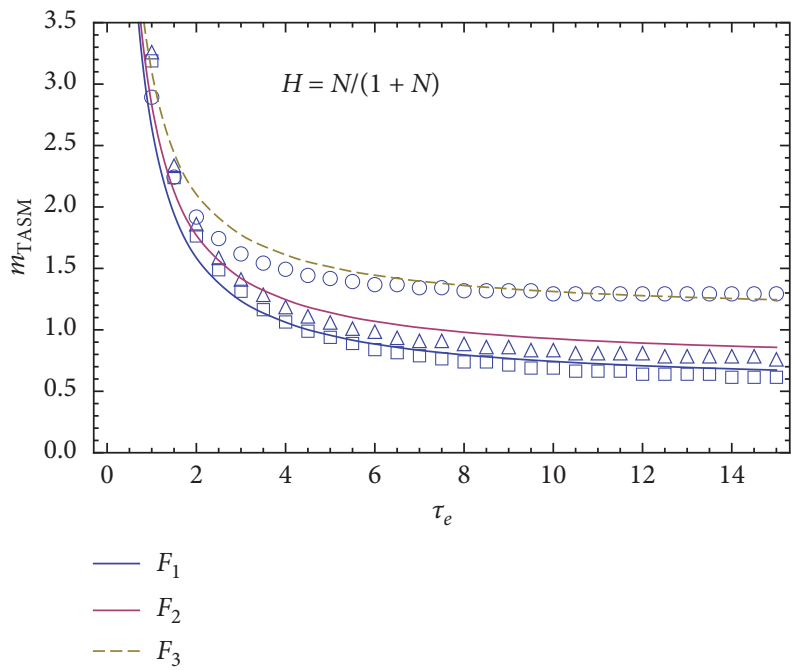

(d)

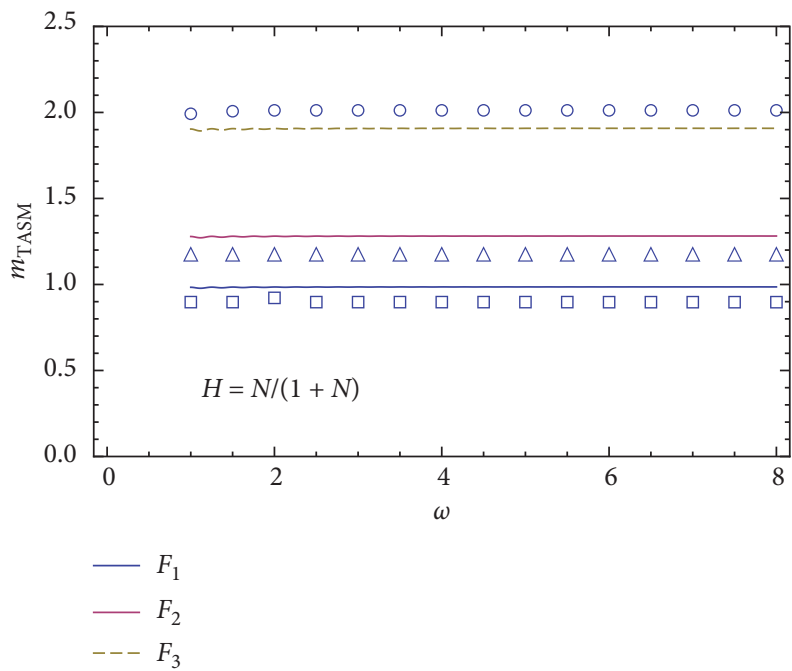

(f)

FIgURE 5: Theoretical and simulated $m_{\mathrm{TASM}}$, in the case of periodic mass release strategy, as a function of $\beta$, as a function of $\tau_{e}$, and as a function of $\omega$. Parameters values are $r=1, \varepsilon=0.5, \varphi=0, \alpha=0.8, \gamma=0.5$, and $(\eta=0.18)$. (a) Parameters values are $\gamma=1, \tau_{s}=0.5, \tau_{e}=30$, and $\omega=1$, (b) parameters values are $\gamma=1, \tau_{s}=0.5, \tau_{e}=30$, and $\omega=1$, (c) parameters values are $\gamma=2, \tau_{s}=4, \omega=5$, and $\beta=15$, (d) parameters values are $\gamma=2, \tau_{s}=4, \omega=5$, and $\beta=15$, (e) parameters values are $\gamma=1, \tau_{s}=5, \tau_{e}=25$, and $\beta=15$, and (f) parameters values are $\gamma=1, \tau_{s}=5$, $\tau_{e}=25$, and $\beta=15$. 


\section{Discussion and Conclusion}

Let us begin our discussion with a comparison between the three release strategies. To do so, two criteria have to be retained, namely: the total number of released sterile males, $M_{\mathrm{SM}}$, and the time required to obtain effective extinction, $\tau_{e}$. These criteria are the most relevant when dealing with the cost of a SIT program. It is worth noting that $M_{\mathrm{SM}}$ and $\tau_{e}$ are closely correlated. However, the way of this correlation depends particularly on the release strategy. Indeed, the total numbers $M_{\mathrm{SM}}^{(S)}, M_{\mathrm{SM}}^{(C)}$ and $M_{\mathrm{SM}}^{(P)}$ released within the three strategies, single, continuous, and periodic, respectively, are given by the following equation:

$$
\begin{aligned}
& M_{\mathrm{SM}}^{(S)}=M_{\mathrm{TASM}}^{(S)}, \\
& M_{\mathrm{SM}}^{(C)}=\tau_{e, c} m_{\mathrm{TASM}}^{(C)}, \\
& M_{\mathrm{SM}}^{(P)}=\tau_{e, p}\left(1+\frac{\varepsilon}{\omega \tau_{e, p}}\left[\sin \left(\omega \tau_{e, p}+\varphi\right)-\sin \varphi\right]\right) m_{\mathrm{TASM}}^{(P)} \approx \tau_{e, p} m_{\mathrm{TASM}}^{(P)},
\end{aligned}
$$

where subscripts $s, c$ and $p$ in $\tau_{e}$ are used to distinguish between effective extinction times relative to the three release strategies. Using theoretical results obtained above for extinction conditions, we can firstly deduce that, for a given couple of intraspecific and interspecific submodels, total numbers of sterile males consumed in continuous and periodic release strategies are almost-equal, for the same extinction time: $\tau_{e, c}=\tau_{e, p}$. Figure 6 illustrates the behaviors of the three total numbers, $M_{\mathrm{SM}}^{(S)}, M_{\mathrm{SM}}^{(C)}$, and $M_{\mathrm{SM}}^{(P)}$ with the extinction time, for the same initial number of the wild population. Particularly, we remark that the total number of sterile males consumed in a single release strategy roughly decays exponentially with the extinction time. However, within a continuous or a periodic release strategy, the total numbers of sterile males used increases linearly with the duration of the extinction process. We should note that results for $M_{\mathrm{SM}}^{(C)}$ and $M_{\mathrm{SM}}^{(P)}$ are valid only for $\tau_{e, c / p}$ well above $\tau_{s}$ since in deriving equations (18) and (23), we have ignored the exponentially decaying parts in the expressions of the wild population numbers $M(t)$, and obviously, such an approximation is valid only for long times in comparison with $\tau_{s}$.

In summary, the paper develops an approach that extends the SIT continuous model for various density-dependent growth rates and interspecific competition functionals. This yields a flexible general model that allows for a substantial simplification of the SIT dynamics and can bias the results in comparison with reality. The model is mathematically interesting because it can produce such a wide range of dynamical behaviors including logistic, strong, and weak Allee effects as well as various response functions for interspecific competition.

Within this general model, analytical and numerical results indicate that eradication of the insect pest is conditional upon the size of the sterile mass release exceeding a critical value. This critical quantity has been determined for various submodels and for three release strategies; single, continuous, and periodic. In all cases,

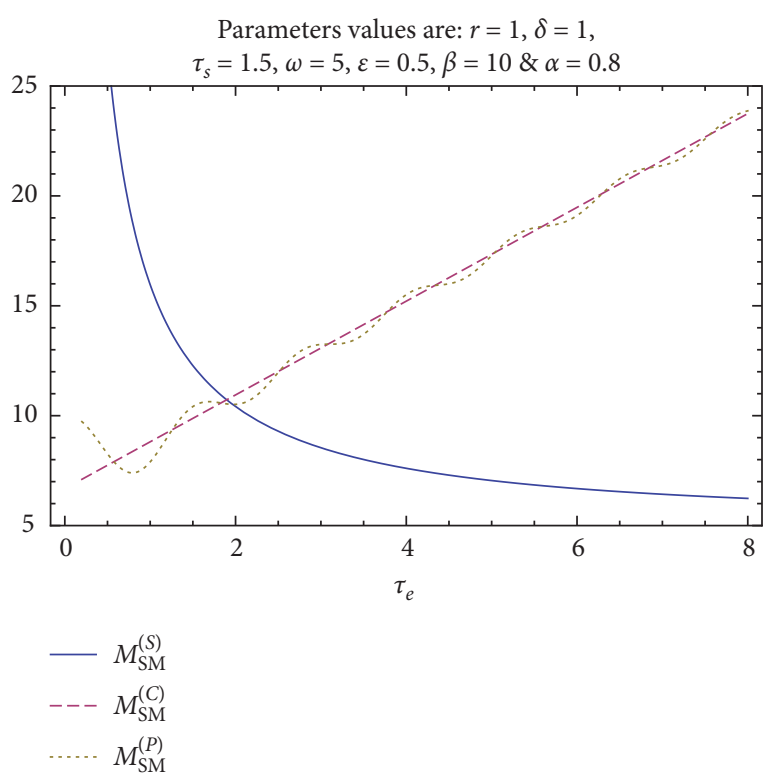

Figure 6: The total number of sterile males, relative to single, continuous, and periodic release strategies, is plotted as functions of the extinction time $\tau_{e}$ for the intraspecific and interspecific submodels $F_{3}$ and $H_{\text {II }}$

numerical computations closely agree with the analytical analysis. The study allowed us to identify the most important parameters which control the choice of release strategies. It also shows that for various release strategies, the sterile weevils life-time is a key parameter in the determination of the TASM. However, the presented model should be seen as a first approximation allowing comparing between idealized sterile male releases in order to get an insight of how these strategies will perform in the field. Indeed, real field mass release programs suffer from many complications that arise from the interaction of several biotic and abiotic factors: weather conditions, host availability, migration of insects, poor timing of release, lack of competitiveness of released weevils, and a multitude of other factors that are crucial to lead a successful release. While it is not possible to include all of these factors in modelling SIT, a great improvement of our model can be gained by introducing controls into the population's dynamics. This can be done a priori in the same manner as with the well-known Lotka-Volterra model, using already existing techniques, such as the speed gradient method and Emel'yanov method. This extension of the model would be the subject of a forthcoming study.

Finally, let us note that the sterile insect technique has a pest density-dependent suppressive action. It has the attribute of increasing efficiency with decreasing target population density. A given chemical or biological insecticide achieves about the same degree of control, whether the pests are abundant or scarce [31]. Therefore, these genetic control methods can be effectively integrated with other density independent and density dependant methods especially when the objective is to manage total populations on an areawide basis. 


\section{Data Availability}

No data were used to support this study.

\section{Conflicts of Interest}

The authors declare that they have no conflicts of interest.

\section{Acknowledgments}

The third author would like to thank his Professors/Scientists Pr. Mohamed Haiour, Pr. Ahmed-Salah Chibi, and Pr. Azzedine Benchettah at Annaba University in Algeria for the important content of masters and $\mathrm{PhD}$ courses in pure and applied mathematics which he received during his studies. Moreover, he thanks them for the additional help they provided to him during office hours in their office about the few concepts/difficulties he had encountered, and he appreciates their talent and dedication for their postgraduate students currently and previously. In addition, the authors gratefully acknowledge Qassim University, represented by the Deanship of Scientific Research, on the material support for this research under the number 6766-alrasscac-bs-20192-2-I during the academic year 1441 AH/2019 AD.

\section{References}

[1] E. F. Knipling, "Possibilities of insect control or eradication through the use of sexually sterile males 1," Journal of Economic Entomology, vol. 48, no. 4, pp. 459-462, 1955.

[2] E. F. Knipling, Screwworm Eradication: Concepts and Research Leading to the Sterile Male Method, pp. 409-418, Smithsonian Institution Publication, Washington, DC, USA, 1959.

[3] V. A. Dyck, J. Hendrichs, and A. S. Robinson, The Sterile Insect Technique, Principles and Practice in Area-wide Integrated Pest Management, Springer, Dordrecht, Netherlands, 2006.

[4] J. L. Rasgon and T. W. Scott, "Wolbachia and cytoplasmic incompatibility in the California culex pipiens mosquito species complex: parameter estimates and infection dynamics in natural populations," Genetics, vol. 165, no. 4, pp. 20292038, 2003.

[5] P.-A. Bliman, D. Cardona-Salgado, Y. Dumont, and O. Vasilieva, "Implementation of control strategies for sterile insect techniques," Mathematical Biosciences, vol. 314, pp. 43-60, 2019.

[6] C. Dufourd and Y. Dumont, "Modeling and simulations of mosquito dispersal. The case of Aedes albopictus," Biomath, vol. 1, no. 2, p. 17, 2012.

[7] C. Dufourd and Y. Dumont, "Impact of environmental factors on mosquito dispersal in the prospect of sterile insect technique control," Computers \& Mathematics with Applications, vol. 66, no. 9, pp. 1695-1715, 2013.

[8] Y. Dumont and J. M. Tchuenche, "Mathematical studies on the sterile insect technique for the chikungunya disease and Aedes albopictus," Journal of Mathematical Biology, vol. 65, no. 5, pp. 809-855, 2012.

[9] J. Li and Z. Yuan, "Modelling releases of sterile mosquitoes with different strategies," Journal of Biological Dynamics, vol. 9, no. 1, pp. 1-14, 2015.

[10] J. Li, L. Cai, and Y. Li, "Stage-structured wild and sterile mosquito population models and their dynamics," Journal of Biological Dynamics, vol. 11, no. sup1, pp. 79-101, 2016.
[11] M. Huang, X. Song, and J. Li, "Modelling and analysis of impulsive releases of sterile mosquitoes," Journal of Biological Dynamics, vol. 11, no. 1, pp. 147-171, 2017.

[12] J. Li, "New revised simple models for interactive wild and sterile mosquito populations and their dynamics," Journal of Biological Dynamics, vol. 11, no. sup2, pp. 316-333, 2017.

[13] W. G. Costello and H. M. Taylor, "Mathematical models of the sterile male technique of insect control," in Mathematical Analysis of Decision Problems in Ecolog (Lect. Notes Biomath., 51, A. Charnes and W. R. Lynn, Eds., Springer-Verlag, Berlin, Germany, pp. 318-359, 1975.

[14] J. C. Flores and R. Beltran, "Gause's exclusion principle revisited: artificial modified species and competition," Journal of Physics A: Mathematical and General, vol. 33, no. 27, pp. 4877-4882, 2000.

[15] H. Barclay and M. Mackauer, "The sterile insect release method for pest control: a density-dependent model," Environmental Entomology, vol. 9, no. 6, pp. 810-817, 1980.

[16] D. R. Miller and D. E. Weidhaas, "Equilibrium populations during a sterile-male release Program1," Environmental Entomology, vol. 3, no. 2, pp. 211-216, 1974.

[17] M. Gyllenberg, J. Hemminki, and T. Tammaru, "Allee effects can both conserve and create spatial heterogeneity in population densities," Theoretical Population Biology, vol. 56, no. 3, pp. 231-242, 1999.

[18] F. Courchamp, L. Berec, and J. Gascoigne, Allee Effects in Ecology and Conservation, Oxford University Press, Oxford, UK, 2008.

[19] J. M. Cushing and J. T. Hudson, "Evolutionary dynamics and strong Allee effects," Journal of Biological Dynamics, vol. 6, no. 2, pp. 941-958, 2012.

[20] G. Livadiotis and S. Elaydi, "General Allee effect in twospecies population biology," Journal of Biological Dynamics, vol. 6, no. 2, pp. 959-973, 2016.

[21] M. A. McCarthy, "The Allee effect, finding mates and theoretical models," Ecological Modelling, vol. 103, no. 1, pp. 99-102, 1997.

[22] R. Lande, S. Engen, and B.-E. Saether, "Optimal harvesting, economic discounting and extinction risk in fluctuating populations," Nature, vol. 372, no. 6501, pp. 88-90, 1994.

[23] T. Stephan and C. Wissel, "Stochastic extinction models discrete in time," Ecological Modelling, vol. 75-76, no. 76, pp. 183-192, 1994.

[24] M. A. Lewis and P. Kareiva, "Allee dynamics and the spread of invading organisms," Theoretical Population Biology, vol. 43, no. 2, pp. 141-158, 1993.

[25] R. R. Veit and M. A. Lewis, "Dispersal, population growth, and the Allee effect: dynamics of the house finch invasion of eastern North America," The American Naturalist, vol. 148, no. 2, pp. 255-274, 1996.

[26] V. Rapisarda, C. Loreto, E. Vitale et al., "Incidence of sharp and needle-stick injuries and mucocutaneous blood exposure among healthcare workers," Future Microbiology, vol. 14, no. 9s, pp. 27-31, 2019.

[27] R. Ragusa, G. Giorgianni, L. Lupo et al., "Healthcare-associated Clostridium difficile infection: role of correct hand hygiene in cross-infection control," Journal of Preventive Medicine and Hygiene, vol. 59, no. 2, pp. E145-E151, 2018.

[28] H. I. Freedman, Deterministic Mathematical Models in Population Ecology, Dekker, NewYork, NY, USA, 1980.

[29] R. May, Stability and Complexity in Model Ecosystems, Princeton University Press, Princeton, NJ, USA, 1974.

[30] J. Hendrichs, A. S. Robinson, J. P. Cayol, and W. Enkerlin, "Medfly areawide sterile insect technique programmes for 
prevention, suppression or eradication: the importance of mating behavior studies," Florida Entomologist, vol. 85, no. 1, pp. 1-13, 2002.

[31] E. F. Knipling, "Sterile insect and parasite augmentation techniques: unexploited solutions for many insect pest problems," Florida Entomologist, vol. 81, no. 1, pp. 134-160, 1998.

[32] F. He and R. I. Alfaro, "White pine weevil (Coleoptera: Curculionidae) attack on white spruce: spatial and temporal patterns," Environmental Entomology, vol. 26, no. 4, pp. 888-895, 1997.

[33] J. R. Faleiro, "Pheromone technology for the management of red palm weevil Rhynchophorus ferrugineus (Olivier) (Coleoptera: rhynchophoridae) a key pest of coconut," Technical Bulletin No.4, ICAR Research Complex for Goa, no. 4, p. 40, 2005.

[34] S.-C. Huang, M. A. Zi-Long, Q. Wi-Quan, L. Chao-Xu, $\mathrm{Y}$. Feng-Yu, and $\mathrm{H}$. Chao-Wen, "The trapping effect of aggregation pheromone for the red palm weevil (rhynchorus ferrugineus (olivier)) and its traps development." China Forestry Science and Technology, vol. 3, pp. 1-9, 2008.

[35] D. K. Butani, "Insect pests of fruit crops and their control, sapota-11," Pesticides Research Journal, vol. 9, pp. 40-42, 1975.

[36] H. S. Salama, F. N. Zaki, and A. S. Abdel-Razek, "Ecological and biological studies on the red palm weevil Rhynchophorus ferrugineus (Olivier)," Archives of Phytopathology and Plant Protection, vol. 42, pp. 392-399, 2009.

[37] A. Martin and S. Ruan, "Predator-prey models with delay and prey harvesting," Journal of Mathematical Biology, vol. 43, no. 3, pp. 247-267, 2001.

[38] N. MacDonald, "Time lags in biological models," volume 27 of Lecture Notes in Biomathematics, Springer, Berlin, Germany, 1978. 